\title{
Capas de a-SiN:H modificadas mediante la irradiación con un láser de excímero
}

\author{
N. Banerji ${ }^{(*)}$, J. Serra $^{(*)}$, S. Chiussi $^{(*)}$, F. Lusquiños $^{(*)}$, B. León $^{(*)}$ y M. Pérez-Amor ${ }^{(*)}$
}

Resumen Utilizando una mezcla de silano/amoníaco o disilano/amoníaco se obtuvieron capas de nitruro de silicio amorfo hidrogenado (a-SiN:H) mediante depósito químico en fase vapor asistido por láser $\mathrm{CO}_{2}$ y $\mathrm{ArF}$, ambos en configuración paralela. Estas capas se sometieron a irradiación con un láser excímero de ArF en atmósfera inerte. Las variaciones, tanto en su composición química como en su índice de refracción y morfología superficial se estudiaron sistemáticamente mediante espectroscopía infrarroja por transformada de Fourier (FTIR), espectroscopía dispersiva de rayos X (EDS), elipsometría y microscopía de fuerzas atómicas (AFM), respectivamente. Las modificaciones observadas involucran por una parte una gran reducción, con los primeros pulsos de irradiación, del hidrógeno enlazado (Si$\mathrm{H}$ y N-H), acompañado por una progresiva incorporación de oxígeno (Si-O y Si-OH) y una reducción de los enlaces $\mathrm{Si}-\mathrm{N}$. Este comportamiento se atribuye a la fotoquímica inducida por la radiación ultravioleta, donde el agua incorporada en las capas, entre sucesivas irradiaciones, desempeña un papel muy importante. La comparación entre las diferentes capas indica que la cantidad de hidrógeno enlazado en las capas y su morfología superficial influyen en la resistencia de las mismas a la oxidación.

Palabras clave: LCVD. Capa delgada. Nitruro de silicio. Irradiación. Láser ArF.

\section{a-SiN:H films modified by ArF excimer laser irradiation}

\begin{abstract}
Hydrogenated amorphous silicon nitride (a:SiN:H) films produced by $\mathrm{CO}_{2}$ and $\mathrm{ArF}$ laser-induced CVD in parallel configuration using silane/ammonia or disilane/ammonia gas mixtures were subjected to room temperature multiple pulse ArF excimer laser irradiations in an inert gas atmosphere. Modifications in their chemical composition, refractive index, and surface morphology were systematically followed up through Fourier-transform infrared spectroscopy (FTIR), energy dispersive $\mathrm{x}$-ray spectroscopy (EDS), single wavelength ellipsometry and atomic force microscopy (AFM), respectively. The observed film modifications involving large reductions in hydrogen $(\mathrm{Si}-\mathrm{H}$ and $\mathrm{N}-\mathrm{H}$ ) with the first few shots accompanied by a progressive incorporation of oxygen ( $\mathrm{Si}-\mathrm{O}$ and $\mathrm{Si}-\mathrm{OH}$ ) and a reduction of $\mathrm{Si}-\mathrm{N}$ bonds that are attributed to UV-induced photochemistry where the atmospheric water incorporated in the films between successive irradiations plays an important role. Comparison among the different films indicated that the extent of bonded hydrogen in the films and their surface morphology influence their oxidation resistance.
\end{abstract}

Keywords: LCVD. Film. Silicon nitride. Irradiation. ArF laser.

\section{INTRODUCCIÓN}

El a-SiN:H presenta una serie de características (eficaz barrera para la difusión, alta constante dieléctrica y buena transmisión óptica) (1) que lo convierten en un material idóneo en múltiples aplicaciones, entre las que podemos nombrar la fabricación de dispositivos dentro de la microelectrónica: como capas finales pasivantes, máscaras

(*) Dpto. Física Aplicada, Univ. de Vigo, Lagoas-Marcosende 9. 36200-Vigo (España). para la oxidación selectiva del silicio (procesos LOCOS), capas aislantes para dispositivos metalnitruro-óxido-silicio (MNOS), etc. Por otra parte, en la última década, dentro del campo de la fabricación de dispositivos de baja temperatura y procesamiento post-depósito, podemos resaltar la utilización de los láseres excímeros en el prerrecocido de capas de nitruro de silicio y recristalización de capas de silicio policristalino, que se utilizan en transistores en capa delgada (TFT) (2).

Con respecto a las modificaciones postdepósito que pueden generarse en estas capas de nitruro de silicio, según se señala en la bibliografía (3), la 
irradiación de a-SiN:H mediante un láser $\mathrm{ArF}$ a una densidad de energía crítica $\left(200 \mathrm{~mJ} / \mathrm{cm}^{2}\right)$ origina una pérdida del nitrógeno incorporado en la capa y la posterior precipitación de silicio cristalino sobre la superficie de la misma. Por otro lado, también es de reseñar que, Takigawa y colaboradores han observado la pérdida de iones nitruro para una densidad de energía menor a $60 \mathrm{~mJ} / \mathrm{cm}^{2}$ (4 y 5).

Tomando como base las investigaciones preliminares sobre irradiación (6), el objetivo de este trabajo es comparar las modificaciones inducidas, en capas de a-SiN:H depositadas con diferentes mezclas de gases y/o fuentes de fotones, tanto a nivel estructural como morfológico.

\section{EXPERIMENTAL}

Se depositaron tres tipos de capas de a:SiNH a $300^{\circ} \mathrm{C}$ en configuración paralela sobre obleas de silicio (100) utilizando la siguiente combinación de láseres y mezcla de gases: láser $\mathrm{CO}_{2}(10,6 \mu \mathrm{m})$ con $\mathrm{SiH}_{4}-\mathrm{NH}_{3}$-Ar, láser de $\mathrm{Ar}(193 \mathrm{~nm})$ con $\mathrm{SiH}_{4}-\mathrm{NH}_{3}-$ Ar y láser $\mathrm{ArF}$ con $\mathrm{Si}_{2} \mathrm{H}_{6}-\mathrm{NH}_{3}$-Ar. Estas muestras se identifican como $\mathrm{CO}_{2}$-silano, ArF-silano y ArF-disilano, respectivamente. Los detalles del sistema experimental se han descrito en trabajos previos (7 y 8).

La irradiación UV de las diferentes capas depositadas se llevó a cabo a temperatura ambiente en atmósfera inerte de argón $\left(\mathrm{P}_{\text {tot }}=100\right.$ Torr $)$, mediante un láser de excímero ArF con pulsos de $20 \mathrm{~ns}$, cuyos fotones tienen una energía de $6,4 \mathrm{eV}$ con una repetición de $1 \mathrm{~Hz}$. Mediante una lente cilíndrica se obtuvo una densidad de energía de $55 \mathrm{~mJ} / \mathrm{cm}^{2}$ (lo cual corresponde a $32,5 \times 10^{6}$ fotones $/ \mathrm{cm}^{2}$ ). Las irradiaciones se realizaron consecutivamente incrementando la duración hasta llegar a un total de 16.000 pulsos. Después de cada irradiación, la muestra se retiró del sistema de vacío y se caracterizó. El espesor típico de las capas depositadas es del orden de $100 \mathrm{~nm}$ y su índice de refracción se midió mediante un elipsómetro $(632,8 \mathrm{~nm})$. La información acerca de la composición de las capas se obtuvo mediante espectroscopía infrarroja por transformada de fourier (FTIR) y espectrometría dispersiva de rayos $\mathrm{X}$ (EDS). Se llevó a cabo un detallado análisis de los espectros infrarrojos en la región comprendida entre $700-1.300 \mathrm{~cm}^{-1}$. Los cambios en la morfología superficial de las capas se observaron por microscopía de fuerzas atómicas (AFM).

\section{RESULTADOS Y DISCUSIÓN}

\subsection{Capas originales}

Los tres tipos de capas de a-SiN:H depositadas tienen unas características generales que pueden atribuirse al tipo de proceso empleado para su depósito. En el primer tipo de proceso, (capa $\mathrm{CO}_{2}$-silano), cuando se utiliza una mezcla de $\mathrm{SiH}_{4}-\mathrm{NH}_{3}$ y para activar la mezcla un láser IR $\left(\mathrm{CO}_{2}, 10,6 \mu \mathrm{m}\right.$ en continuo), tiene lugar un calentamiento del gas, alcanzándose valores lo suficientemente elevados para que se produzca la pirólisis del silano $\left(\mathrm{E}_{\mathrm{act}}=\right.$ $52 \mathrm{kcal} / \mathrm{mol})$, mientras el amoníaco $\mathrm{NH}_{3}\left(\mathrm{E}_{\mathrm{act}}\right.$ $=92 \mathrm{kcal} / \mathrm{mol}$ ) reacciona con los productos descompuestos en la fase gaseosa, formando aminosilanos intermedios $\left(\mathrm{Si}_{\mathrm{x}} \mathrm{N}_{\mathrm{y}} \mathrm{H}_{\mathrm{z}}\right)$ (9). Estas capas, en general, tienen un alto índice de refracción (Tabla I) y un alto contenido de hidrógeno y silicio comparado con el nitrógeno (10), como confirman los espectros de infrarrojo (Fig.1, espectro I. $a$ ).

El segundo proceso (capa ArF-silano) lleva implícito un mecanismo de fotólisis; la fuente de activación de la mezcla $\left(\mathrm{SiH}_{4}-\mathrm{NH}_{3}\right)$ es un láser excímero de ArF, donde el primer paso es la fotodisociación del amoníaco, el cual presenta una sección eficaz de absorción $\sigma=1 \times 10^{-17} \mathrm{~cm}^{2}$, mucho mayor que la del silano $\left(2 \times 10^{-21} \mathrm{~cm}^{2}\right)$. Posteriormente, el silano reacciona con los productos de la descomposición del amoníaco para producir aminosilanos (9). Estas capas tienen un valor del índice de refracción $n=1.8$, típico de un nitruro de silicio (11) y son ricas en hidrógeno y nitrógeno, lo cual es evidente por la relativa abundancia de los grupos $\mathrm{N}-\mathrm{H}(\mathrm{N}-\mathrm{H}$ y $\mathrm{NH}_{2}$ ) y $\mathrm{Si}-\mathrm{H}\left(\mathrm{Si}-\mathrm{H}\right.$ y $\left.\mathrm{Si}-\mathrm{H}_{2}\right)$ que se observan en el espectro infrarrojo (Fig.1, espectro II. $a$ ).

En el tercer proceso (capa ArF-disilano) se ha utilizado como fuente de activación también un láser $\mathrm{ArF}$, pero la mezcla es, ahora, $\mathrm{Si}_{2} \mathrm{H}_{6}-\mathrm{NH}_{3}$; en este caso, la sección de absorción del disilano $(2 \times$ $10^{-18} \mathrm{~cm}^{2}$ ) es tan importante como la del amoníaco y sus productos reaccionan produciendo capas con un menor contenido de hidrógeno y ricas en silicio (12). El espectro infrarrojo de este tipo de capas se representa en la figura1, espectro III. $a$. El valor de su índice de refracción confirma este hecho.

Las variaciones entre los tres diferentes tipos de capas, a nivel de su estructura química, debido a su diferente composición, queda reflejado en las posiciones de los picos $\mathrm{Si}-\mathrm{H}$ y Si-N stretching los cuales se desplazan hacia números de onda mayores a medida que se incrementan los enlaces de $\mathrm{N}-\mathrm{H}$ en la red amorfa. Este es el caso de la capa ArF-silano, donde las bandas $\mathrm{Si}-\mathrm{N}$ y Si-H stretching aparecen $\mathrm{a} \approx 834$ y $2.152 \mathrm{~cm}^{-1}$, respectivamente, mientras en las otras dos capas, ricas en silicio, la posición de estos picos está cerca de 830 y $2.115 \mathrm{~cm}^{-1}$, respectivamente. Argumentos basados en la electronegatividad de los átomos constituyentes sugiere que la formación de enlaces N-H o Si-H en el lugar de Si-N conduce a un desplazamiento opuesto en la frecuencia del enlace Si-N (13).

En cuanto a la caracterización topográfica de la superficie de las capas antes de irradiar (Figs.2 $a, b$ 
TABLA I.- Resultados de las diferentes caracterizaciones realizadas para cada una de las muestras: Elipsometría $(n)$, perfilometría (espesor), fuerzas atómicas (AFM) y espectroscopía de dispersión de rayos X (EDS).

TABLE I.- Results of the different characterizations carried out or each one of the samples: Ellipsometry (n), perfilometry (thickness), atomic forces (AFM) and X-ray dispersive energy espectroscopy (EDS).

\begin{tabular}{|c|c|c|c|c|c|c|}
\hline Muestra & $\begin{array}{c}\text { Mezcla } \\
\text { de } \\
\text { gases }\end{array}$ & $\begin{array}{c}\text { Velocidad de } \\
\text { depósito } \\
\AA\end{array}$ & $\begin{array}{c}\text { Índice de } \\
\text { refracción } \\
(\mathrm{n})\end{array}$ & $\begin{array}{c}\text { AFM } \\
\text { rugosidad } \\
(\mathrm{R}) \mathrm{nm}\end{array}$ & $\begin{array}{c}\Delta \mathrm{R} \\
(*)\end{array}$ & $\begin{array}{c}\text { EDS } \\
\Delta \% \mathrm{~N} \\
(* *)\end{array}$ \\
\hline $\mathrm{CO} 2$ silano & $\mathrm{SiH}_{4} / \mathrm{NH}_{3}$ & $2.5 \times 10^{-1}$ & 2.8 & 0.343 & 26.59 & 0.716 \\
\hline ArF silano & $\mathrm{SiH}_{4} / \mathrm{NH}_{3}$ & 2.3 & 1.8 & 2.468 & 6.833 & 0.364 \\
\hline ArF disilano & $\mathrm{Si}_{2} \mathrm{H}_{6} / \mathrm{NH}_{3}$ & 3.0 & 2.3 & 9.624 & 0.579 & 0.188 \\
\hline
\end{tabular}

(*) Variación de la rugosidad en las capas: $\Delta R=\frac{R_{\text {irradiada }}-R_{\text {sin-irradiar }}}{R_{\text {sin-irradiar }}}$

Variation of the roughness in the films: $\Delta R=\frac{R_{\text {irradiated }}-R_{\text {as-deposited }}}{R_{\text {as-deposited }}}$

(**) Variación del contenido de nitrógeno en las capas: $\Delta \% N=\frac{\% N_{\text {sin-irradiar }}-\% N_{\text {irradiada }}}{\% N_{\text {sin-irradiar }}}$

Variation of nitrogene content in the films: $\Delta \% N=\frac{\% N_{\text {as-deposited }}-\% N_{\text {irradiated }}}{\% N_{\text {as-deposited }}}$

y c) ésta indica que la capa $\mathrm{CO}_{2}$-silano es la que presenta un tamaño de partícula menor y una mayor homogeneidad, seguida de la capa ArF-silano y, por último, la ArF-disilano. Esto puede estar asociado al incremento en el ritmo de depósito de las capas (0.25, 2.3 y $3 \AA / s$, respectivamente) lo cual conduce a la formación de un mayor tamaño de grano y rugosidad.

\subsection{Capas irradiadas}

Durante el proceso de irradiación UV se han observado profundas modificaciones, como queda reflejado en los espectros infrarrojos (Fig. 1 I, II, III). Después de los primeros pulsos, se observa en todas las capas una rápida reducción del área de los picos $\mathrm{Si}-\mathrm{H}$ y $\mathrm{N}-\mathrm{H}$ y un aumento de la anchura a media altura (FWHM) del pico de Si-N, así como un desplazamiento de éste $\left(\approx 15 \mathrm{~cm}^{-1}\right)$ hacia mayores números de onda. Se atribuye este ensanchamiento a la incorporación de grupos de $\mathrm{Si}-\mathrm{OH}$ producidos mediante un mecanismo que tiene lugar cuando la radiación UV induce reacciones con el agua absorbida en la capa.

Con las sucesivas irradiaciones (Fig. 1), se observa la aparición, ya clara, de un pico asignado al Si-O stretching $\left(1.060 \mathrm{~cm}^{-1}\right)$ en forma de un hombro en la banda del enlace Si-N. Este pico tiene su aparición a diferentes tiempos de irradiación, dependiendo de la capa: 16 pulsos en la $\mathrm{CO}_{2}$-silano, 64 pulsos en la ArF-silano y 256 pulsos en la ArFdisilano. Con el objeto de estudiar más a fondo este proceso, se ha llevado a cabo la deconvolución de la zona entre 700 y $1.300 \mathrm{~cm}^{-1}$, considerando las siguientes contribuciones: enlaces $\mathrm{Si}-\mathrm{O}$ con modos de vibración $1.060 \mathrm{~cm}^{-1}$ (stretching) y $800 \mathrm{~cm}^{-1}$ (bending), Si-N (stretching) $840 \mathrm{~cm}^{-1}, \mathrm{Si}-\mathrm{OH}$ (bending) $940 \mathrm{~cm}^{-1}$ y Si-H (bending) $880 \mathrm{~cm}^{-1}$. El pico localizado a $1.178 \mathrm{~cm}^{-1}$ puede asociarse a enlaces $\mathrm{N}-\mathrm{H}$ (bending) o Si-O (transverse) (14 y 15). La evaluación de la absorbancia de las principales bandas (Si-O y Si-N) (Fig.3) indica que el nitrógeno (oxigeno) enlazado disminuye (aumenta) con los primeros pulsos de irradiación del láser. Estos resultados concuerdan con el microanálisis realizado por EDS (Tabla I), el cual indica una menor cantidad de nitrógeno en todas las capas después de ser irradiadas. También se ha podido observar que la variación relativa en porcentaje atómico de nitrógeno es mayor para la capa de $\mathrm{CO}_{2}$-silano, seguida de la de ArF-silano y, por último, la de ArF-disilano.

Finalmente, los cambios topográficos que sufre la superficie de las capas con la irradiación (16.000 pulsos) se muestran en la figura $2 a-i, b-i$ y $c-i$, donde se puede observar un aumento del tamaño de 


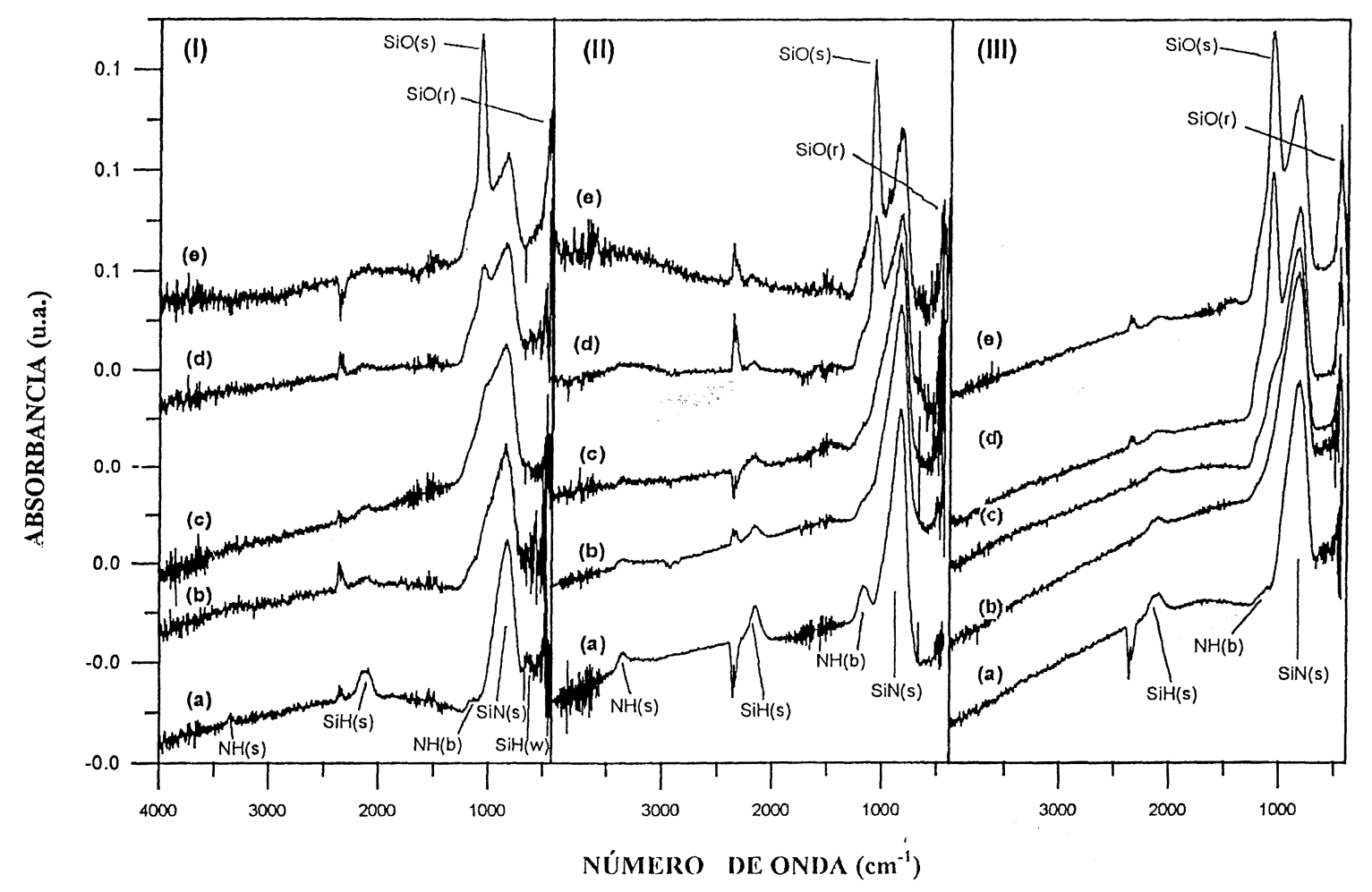

FIG. 1. - Espectros de FTIR de las diferentes capas de a-SiN:H y su evolución a lo largo del proceso de irradiación UV: (I) capa. $\mathrm{CO}_{2}$ silano: $a$ ) sin irradiar; $b$ ) 16 pulsos, $c$ ) 64 pulsos, $d$ ) 256 pulsos, $e) 16.000$ pulsos. (II) capa. ArF silano: $a$ ) sin irradiar, $b$ ) 16 pulsos $c$ ) 64 pulsos $d$ ) 512 pulsos, e) 16.000 pulsos y (III) capa. ArF disilano: $a$ ) sin irradiar, $b$ ) 16 pulsos, c) 256 pulsos, d) 1.024 pulsos y $e) 16.000$ pulsos.

FIG. 1.- Representative FTIR spectra of the SiN film evolution under a progressive ArF laser irradiation: (I) CO2 silane film. a) as-deposited, b) 16 pulses, c) 64 pulses, d) 256 pulses, e) 16.000 pulses. (II) ArF silane film. a) as-deposited, b) 16 pulses, c) 64 pulses, d) 512 pulses e) 16.000 pulses and (III) ArF disilane film. a) as-deposited, b) 16 pulses, c) 256 pulses, d) 1.024 pulses and e) 16.000 pulses.

grano en todas las capas, lo cual es similar a lo que se observa en el proceso de recocido de silicio amorfo mediante láser de excímero (16). La variación relativa del valor de la rugosidad y del tamaño de grano son mayores en la capa de $\mathrm{CO}_{2}$-silano, seguida por ArF-silano y ArF-disilano.

\subsection{Mecanismo de modificación de las capas}

Dentro del proceso de irradiación están presentes dos fenómenos diferentes: el primero es fotolítico; tiene lugar por el hecho de que los fotones emitidos por la radiación láser $\operatorname{ArF}(6.4 \mathrm{eV})$ son suficientemente energéticos para fotodisociar los enlaces presentes en la capa de a-SiN:H. El segundo es un fenómeno pirolítico que se origina por un efecto de retroalimentación dinámica debido a un acoplamiento de la conductividad térmica y óptica a través de la dispersión electrón-fonón originando un rápido incremento de la temperatura de la superficie de la capa. Teniendo en cuenta las condiciones de esta investigación, se puede considerar, por una parte, que los coeficientes de difusión del calor permanecen constantes durante el período de irradiación, que las aproximaciones de la ecuación de difusión del calor son válidas y que se irradia con longitudes de onda por encima del ancho de banda prohibido, por tanto el calentamiento de la superficie es de $\sim 250{ }^{\circ} \mathrm{C}$ durante los $20 \mathrm{~ns}$ del pulso. Este calentamiento puede alterar la morfología microscópica superficial e influir en las interacciones capa -radiación UV (17).

Para las capas consideradas aquí, la oxidación puede verse limitada por disponibilidad de los átomos de silicio reactivos, los cuales pueden generarse por la ruptura de enlaces $\mathrm{Si}-\mathrm{H}$ y $\mathrm{N}-\mathrm{H}$. El oxígeno de la capa puede generarse inicialmente por la fotodisociación del agua absorbida y procesos fotolíticos pueden producir radicales de oxígeno altamente reactivo. Considerando la menor energía del enlace de $\mathrm{Si}-\mathrm{H}$ comparado con $\mathrm{N}-\mathrm{H}$, las capas EN donde prevalecen los grupos $\mathrm{Si}-\mathrm{H}\left(\mathrm{CO}_{2}\right.$-silano) muestran una ruptura más rápida de enlaces. 


\section{Capas sin-irradiar}

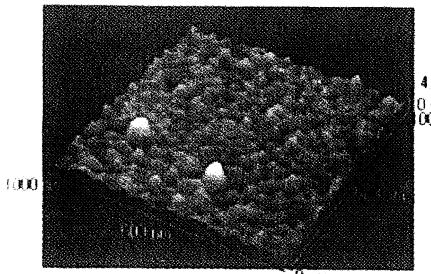

(a)

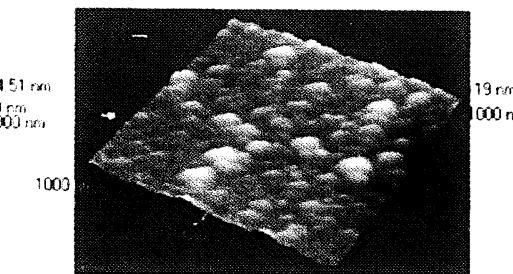

(b) ${ }^{\text {nan }}$ min

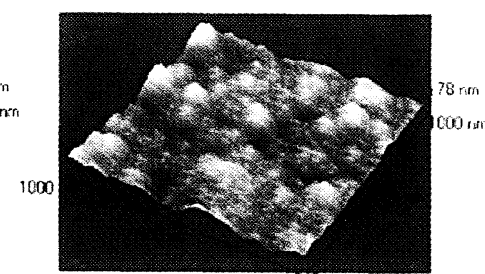

(c)

\section{Capas irradiadas}

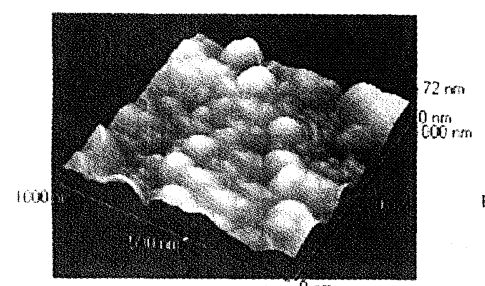

$(\mathrm{a}-1)$

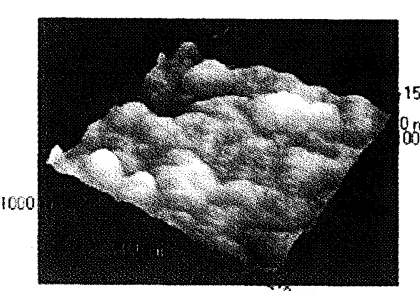

$(\mathrm{b}-1)^{0 \mathrm{~mm} / \mathrm{mm}}$

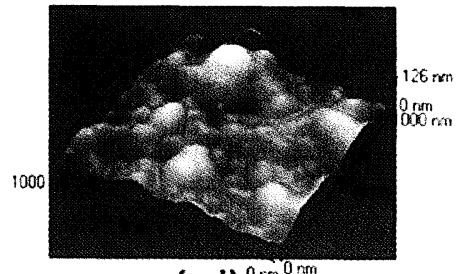

$(c-i)^{0 \mathrm{~mm} 0 \mathrm{rm}}$

FIG. 2.- Imágenes AFM de la superficie de las capas sin-irradiar e irradiadas (16.000 pulsos): (a, a-i) $\mathrm{CO}_{2}$ silano, (b, b-i) ArF silano, (c, c-i) ArF disilano, respectivamente.

FIG. 2.- AFM images of as-deposited and laser-irradiated (16.000 pulses) sample surface: (a, a-i) $\mathrm{CO}_{2}$ silane, $(b, b-i)$ ArF silane, (c, $\left.c-i\right)$ ArF disilane, respectively.

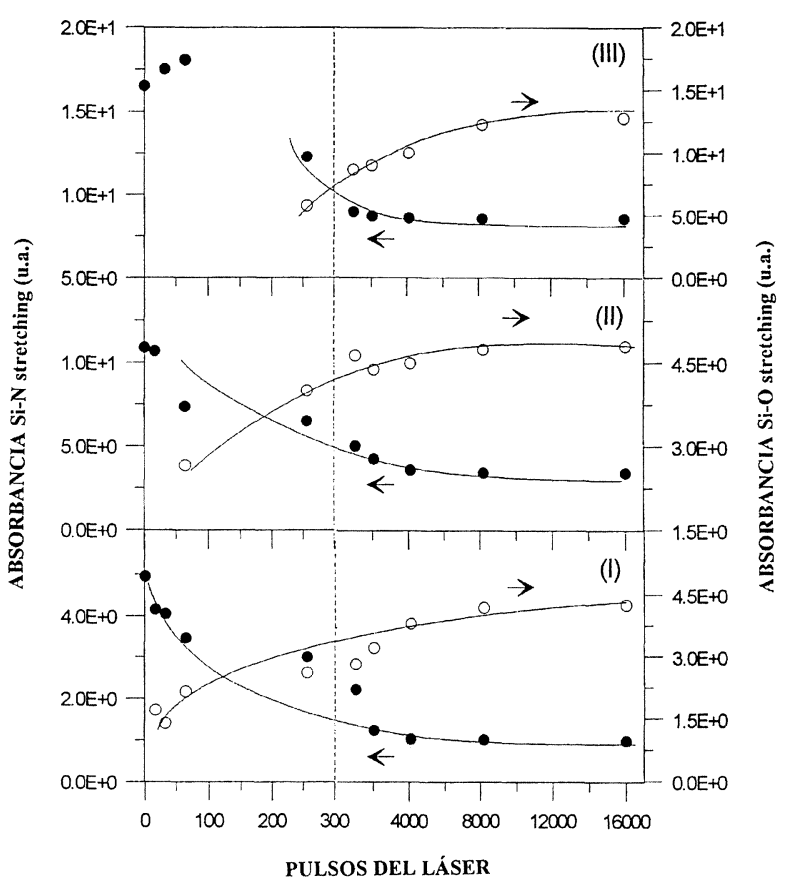

FIG. 3.- Absorbancia de las principales bandas de vibración asignadas a los enlaces $\mathrm{Si}-\mathrm{N}$ stretching (-) y Si-O stretching $(\bigcirc)$ en función del número de pulsos con que fueron irradiadas: (I) $\mathrm{CO}_{2}$ silano;

(II) ArF silano y (III) ArF disilano.

FIG. 3.- Absorbance of the main deconvoluted bands assigned to $\mathrm{Si}-\mathrm{N}$ stretching (O) and $\mathrm{Si}-\mathrm{O}$ stretching $(O)$ vibrations as a function of the $U V$ photon irradiation: (I) $\mathrm{CO}_{2}$ silane film; (II) $\mathrm{ArF}$ silane film and (III) ArF disilane film.
Mientras predominen grupos $\mathrm{N}-\mathrm{H}$, la oxidación es comparativamente más lenta. La capa ArF-disilano, con una menor cantidad de enlaces de hidrógeno, se oxida más lentamente, a pesar de que con los primeros pulsos incorpora más grupos $\mathrm{Si}-\mathrm{OH}$ (debido a la ruptura de enlaces $\mathrm{Si}-\mathrm{Si}$ ) que la capa $\mathrm{ArF}$ silano.

\section{CONCLUSIONES}

Por una parte, aunque todas las capas sometidas a la irradiación UV sufren un proceso de oxidación, se ha observado que este proceso se ve afectado por la estructura composicional y morfológica inicial que presenta cada capa. Así, la capa depositada mediante un proceso pirolítico, $\mathrm{CO}_{2}$-silano, muestra una mayor modificación en su estructura química y en su morfología, seguida por la depositada fotolíticamente ArF-silano y finalmente la capa ArF-disilano. Este proceso de oxidación combina, por una parte, mecanismos fotolítico y pirolítico y envuelve la descomposición de enlaces Si-H, N-H y Si-N y la formación de enlaces $\mathrm{Si}-\mathrm{O}$ y $\mathrm{Si}-\mathrm{OH}$.

\section{Agradecimiento}

Los autores agradecen a la Xunta de Galicia y a la Universidad de Vigo la financiación parcial de esta investigación. 


\section{REFERENCIAS}

(1) Shimizu, K., NaKamura, K., Higasimoto, M., Sugiura, O. and Matsumura, M. Jpn. J. Appl. Phys. 32 (1993) 452.

(2) Belyi, V.I. et al, Silicon Nitride in Electronics, Elsevier Science Publishers B.V., Amsterdam, 1988.

(3) Nakamae, K., Kurosawa, K., Takigawa, Y., Sasaki, W., Izawa, Y. and OKuda, M. Jpn. J. Appl. Phys. 34 (1995) L1482.

(4) Takigawa, Y., Sasaki, W., Kato, Y., OKuda, M. Nuclear Instruments and Methods in Physics Research, B, 116, (1996), 211.

(5) Nakamae, K., Kurosawa, K., Ohmukai, H., Katto, M., OKuda, M., Sasaki, W., Nozawa, S. and Igarashi, T. Nuclear Instr. and Meth. B 91 (1994) 659.

(6) Serra, J., Parada, E.G., González, P., Fernández, D., Chiussi, S., Pou, J., LeÓn, B., PÉreZ -Amor, M. Surface Coatings and Technology 80 (1996) 211.

(7) Serra, J., Szörényi, T., Fernández, D., González, P., García, E., Pou, J., LeÓN, B., Pérez-Amor, M. Journal of Non-Crystalline Solids 187 (1995) 35.
(8) Parada, E. G., González, P., Serra, J., León, B., PÉrez-Amor, M., Flicstein, J. and Devine, R.A.B. Appl. Surf. Sci. 86 (1995) 294.

(9) Herman, I. Chem. Rev. 89, (1989) 1323.

(10) Pan, E. T. S., Flint, J. H., Adler, D., Haggerly, J. S. J. Appl. Phys. 61 (9) 1987.

(11) Jasinski, J.M., Mayerson, B.S., Nguyen, T. N. J. Appl. Phys. 61 (1987) 431.

(12) Henda, R., Laanab, L., Scheid, E., Fourmeaux, R. Jpn. J. Appl. Phys. 34 (1995) L 437.

(13) Lucovsky, G., Tsu, D. V., Vac. J. Sci. technol. A5 (4) (1987) 2231.

(14) KIRK, C. T. Phys. Rev. B (1988) 1255.

(15) Watanabe, H., Miki, H., Sugai, S., Kawasaki, K., KioKa, T. Jpn. J. Appl. Phys. 33 (1994), 4491.

(16) BÄUERLE, D. Chemical Processing with Lasers, SpringerVerlag, 1986.

(17) Aussenegg, F. R., Leitner, A., LipPitsch, M. E. Surface Studies with Lasers, Springer Ser. Chem. Phys. 33, 1983. 